\title{
Erratum to: Psychosocial Determinants of Mistimed and Unwanted Pregnancy: The Hamamatsu Birth Cohort (HBC) Study
}

\author{
Shun Takahashi $\cdot$ Kenji J. Tsuchiya $\cdot$ \\ Kaori Matsumoto · Katsuaki Suzuki • \\ Norio Mori · Nori Takei $\cdot$ The HBC Study Team
}

Published online: 20 June 2012

(C) Springer Science+Business Media, LLC 2012

\section{Erratum to: Matern Child Health J \\ DOI 10.1007/s10995-011-0881-y}

In the article "Psychosocial Determinants of Mistimed and Unwanted Pregnancy: The Hamamatsu Birth Cohort (HBC) Study" by S. Takahashi et al., the Method section contained an error in the text. In the first paragraph, under the subheading of Measurement, the fourth and fifth sentences should read as follows: "Among those who answered negatively to this question, a second question was posed-namely, was the pregnancy wanted or not? If $\underline{n}$, the parturients were categorized into the UWP group."

The online version of the original article can be found under doi:10.1007/s10995-011-0881-y.

S. Takahashi · K. J. Tsuchiya ( $₫) \cdot$ K. Suzuki · N. Takei Department of Child Development, United Graduate School of Child Development at Hamamatsu, Hamamatsu 431-3192, Japan

e-mail: tsuchiya@hama-med.ac.jp

S. Takahashi $\cdot$ K. J. Tsuchiya $\cdot$ K. Matsumoto $\cdot$ K. Suzuki ·

N. Mori $\cdot$ N. Takei

Research Center for Child Mental Development, Hamamatsu

University School of Medicine, Hamamatsu, Japan

S. Takahashi

Shizuoka Center for Child and Family Welfare, Shizuoka, Japan

N. Mori

Department of Psychiatry, Hamamatsu University School

of Medicine, Hamamatsu, Japan

N. Takei

Division of Psychological Medicine, Institute of Psychiatry, King's College London, De Crespigny Park, London SE5 8AF, UK 